\title{
Finite-source dye-diffusion thermal transfer for doping and color integration of organic light-emitting devices
}

\author{
Chung-chih $\mathrm{Wu},{ }^{\text {a) }}$ Cheng-chung Yang, Hsin-hua Chang, ${ }^{\text {b) }}$ Chieh-Wei Chen, \\ and Cheng-Chung Lee ${ }^{\text {b) }}$ \\ Department of Electrical Engineering and Graduate Institute of Electro-optical Engineering, National \\ Taiwan University, Taipei, Taiwan 10617, Republic of China
}

(Received 17 January 2000; accepted for publication 30 May 2000)

\begin{abstract}
An effective process of performing controllable doping of polymer films in organic light-emitting devices is reported. In this approach, a film to be doped is brought into direct contact with a dye-dispersed polymer donor film to permit direct dye-diffusion thermal transfer. Theoretical and experimental studies indicate that this doping process can be modeled by Fick's diffusion theory and that a desired dopant distribution may be obtained in a single transfer step by adjusting the diffusion conditions. Doped-polymer light-emitting devices made by this process exhibited the same device characteristics as those by the conventional blending process. Along with patterned color donor plates, we also demonstrated multicolor devices of arbitrary patterns over large areas with a single thermal transfer step. (c) 2000 American Institute of Physics. [S0003-6951(00)03829-8]
\end{abstract}

Organic light-emitting devices (OLEDs) based on molecular organic materials or on polymer materials have been extensively studied because of their various merits suitable for flat-panel display applications. ${ }^{1,2}$ The capability of solution processing of polymers leads to manufacturing advantage for large-area coating. However, it is difficult to integrate multicolor polymer materials or devices for full-color displays by conventional coating and sequential photolithography. ${ }^{3,4}$ To resolve this issue, printing approaches have been aggressively pursued recently. For instance, ink-jet printing has been used to deposit directly patterned polymers or to introduce color-tuning dye patterns into/onto continuous buffer polymer layers. ${ }^{5-8}$ However, inkjet printing is, in general, a sequential process and might have a limit for substrate throughput. ${ }^{9}$ Alternatively, a largearea thermal transfer process was recently proposed for color patterning of polymer layers in OLEDs. ${ }^{9}$ Owing to a transfer mask inserted between the donor and the receiver films, this process involves several mechanisms: the sublimation of dye molecules from the donor film, travel of dye molecules to the surface of the receiver film, and the diffusion of dye molecules into the receiver film. Interaction of all these mechanisms has led to difficulty in controlling profiles of dye concentration and to complication of processing steps. For instance, long periods of annealing may be required to redistribute dye dopants in the receiver film after the transfer process.

In this letter, we report an effective transfer process for performing controllable doping of polymer films in OLEDs. In this process, the polymer receiver film is placed in direct contact with the dye-dispersed polymer donor film to permit direct dye-diffusion thermal transfer (Fig. 1). Theoretical and experimental studies of this doping process show that it can be modeled by Fick's diffusion theory under impermeable film-substrate boundary conditions. We, therefore, call this

\footnotetext{
${ }^{a}$ Electronic mail: chungwu@cc.ee.ntu.edu.tw

b) Institute of Optical Sciences, National Central University, Chung-Li, Taiwan 32054, Republic of China.
}

process finite-source dye-diffusion thermal transfer (FSD2T2).

In performing FS-D2T2, a pressure (typically, 30 $\left.\mathrm{g} / \mathrm{cm}^{2}\right)$ is applied on top of the receiver plate to ensure intimate contact with the source film. If the dye diffusion at an elevated temperature obeys Fick's diffusion theory, under the impermeable boundary conditions at two film-substrate interfaces, the spatial distribution of dye concentration $C(x, t)$ at time $t$ is given by an infinite sum of error functions: $:^{10}$

$$
\begin{aligned}
C(x, t)= & \frac{1}{2} C_{0} \sum_{n=0}^{\infty}\left\{\operatorname{erf}\left(\frac{-x-2 n\left(d_{S}+d_{R}\right)}{2 \sqrt{D t}}\right)\right. \\
& \left.+\operatorname{erf}\left(\frac{x+2 d_{S}+2 n\left(d_{S}+d_{R}\right)}{2 \sqrt{D t}}\right)\right\} \\
& +\frac{1}{2} C_{0} \sum_{n=1}^{\infty}\left\{\operatorname{erf}\left(\frac{-x+2 n\left(d_{S}+d_{R}\right)}{2 \sqrt{D t}}\right)\right. \\
& \left.+\operatorname{erf}\left(\frac{x+2 d_{S}-2 n\left(d_{S}+d_{R}\right)}{2 \sqrt{D t}}\right)\right\},
\end{aligned}
$$

where $x$ represents the depth into the receiver, $C_{0}$ the initial dye concentration in the source, $d_{S}$ and $d_{R}$ the thickness' of the source and the receiver layers, and $D$ denotes the diffusion coefficient of dyes in the matrix. Here, for mathematical and analytical simplicity, we have assumed that the source and the receiver matrices are of the same composition and that the temperature and $D$ are constant throughout both layers. The behavior of $C(x, t)$ versus normalized diffusion length $\left(\sqrt{D t / d_{R}{ }^{2}}\right)$ reveals that dyes originally in the source basically undergo a redistribution process. Since dye redistribution is a self-limiting process, there would be no need for tight control of diffusion conditions to prevent overdoping or consequent concentration quenching in the receiver as long as $C_{0}$ is chosen to match the desired final equilibrium concentration in the receiver. Also, shallow-to-flat profiles into the receiver may be obtained by varying the diffusion length, a feature maybe useful in different device designs. 


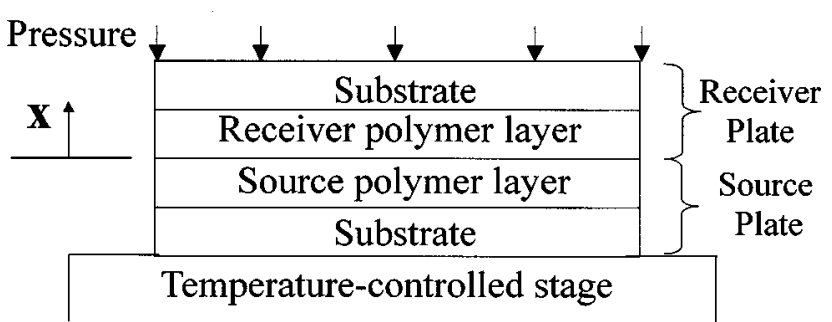

FIG. 1. Experimental arrangement for finite-source dye-diffusion thermal transfer.

To verify these concepts, we performed a series of D2T2 experiments based on the deep-blue-emitting polymer poly $(N$-vinylcarbazole) (PVK). Since PVK only transports holes, to prepare films for OLED fabrication, $\sim 25 \mathrm{wt} \%$ of electron-transport molecules 2-(4-biphenyl)-5-(4-tert-butylphenyl)-1,3,4-oxadiazole (PBD) was added to the PVK matrix by blending. ${ }^{5,6}$ Results presented in this letter are for source and receiver layers of this same matrix composition and the same thickness $(\sim 1000 \AA)$. The concept of FSD2T2, however, can be extended to different source and receiver matrices by taking into account the concentration partition effect. ${ }^{11,12}$ Dyes such as perylene (blue), coumarin 6 (C6, green), and nile red (orange-red) were used in this study. Films were coated onto quartz substrates for photoluminescence (PL) and absorption measurements All the thermal transfer experiments, device fabrication, and characterization were performed in air.

Figure 2 shows the growth with time of absorption and PL intensities associated with the dye (perylene) diffused into the receiver layer at $130^{\circ} \mathrm{C}$. The initial dye concentration in the source $\left(C_{0}=2.1 \mathrm{wt} \%\right)$ was chosen to give nearly optimal equilibrium concentration for PL in the receiver. Ac-
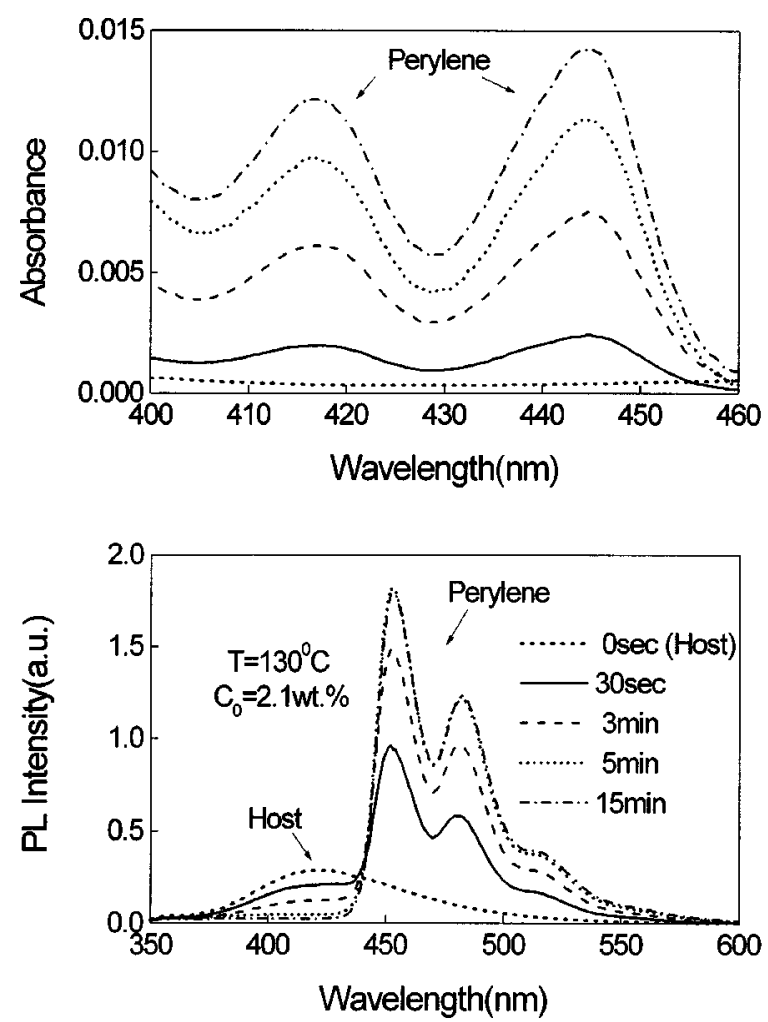

(b)

FIG. 2. (a) Absorption spectra and (b) photoluminescence spectra of dyediffused films vs diffusion time.

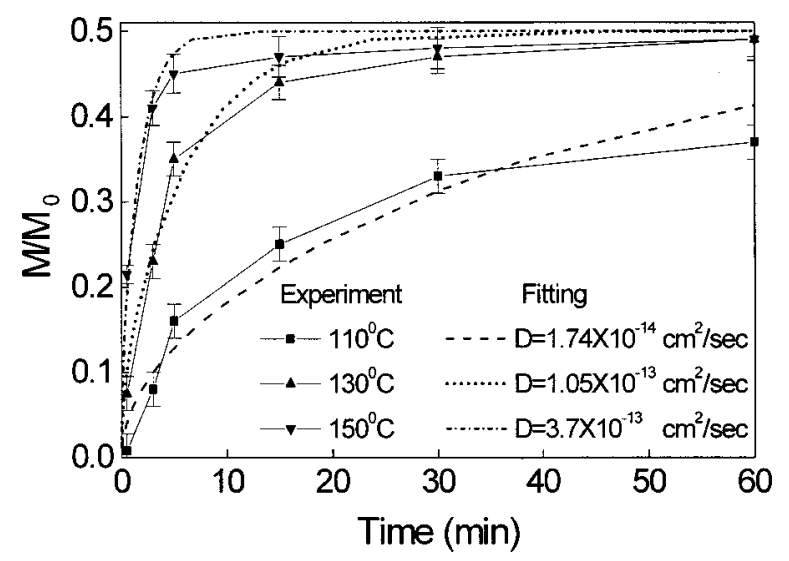

FIG. 3. Ratio of dyes transferred vs diffusion time at different temperatures.

cording to Beer's law, the ratio of the amount of dyes diffusing into the receiver $(M)$ to the initial amount of dyes in the source layer $\left(M_{0}\right)$ can be obtained by dividing the associated absorbance intensities. Results of $M / M_{0}$ for various diffusion conditions are plotted in Fig. 3, in which the leastsquare fitting of experimental data from Fick's theory is also presented. With $D$ as the only parameter, the fitting reasonably follows the trend of experimental data. The extracted $D$ ranges from $10^{-12}$ to $10^{-15} \mathrm{~cm}^{2} / \mathrm{s}$, depending on the dye and the temperature $\left(100-150{ }^{\circ} \mathrm{C}\right)$ used. The self-limiting effect is explicated in the saturation of $M / M_{0}$ versus time. PL results show that within a few minutes (or tens of seconds at even higher diffusion temperature), the diffused dye profile is deep enough to completely quench the luminescence from the host, consistent with absorption results. The PL spectrum and intensity for the completely transferred receiver film are basically the same as those of a conventional dye-blended film with a similar dye concentration, showing no indication of degradation. Fig. 4 compares device characteristics of OLEDs using perylene-diffused and perylene-blended PVK:PBD films with similar dye concentrations as the active emission layer. Both have the device structure of glass indium-tin-oxide (ITO) anode/hole-injection layer $(\sim 300$ $\AA) /$ active emission layer $(\sim 1000 \quad \AA) / \mathrm{Mg}: \mathrm{Ag} \quad(10: 1)$ cathode/Ag protection layer, where the conducting polymer polyethylene dioxythiophene/polystyrene sulphonate (PEDT/ PSS, Bayer Corp.) was used as the hole-injection layer. Within our experimental variation, both types of devices gave nearly the same characteristics with an external quantum efficiency of $\sim 0.8 \%$. From these results, we conclude

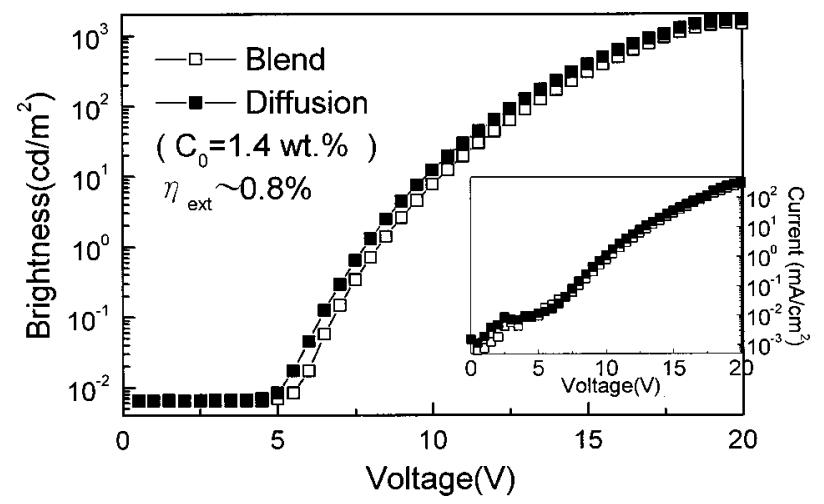

FIG. 4. Comparison of device characteristics of OLEDs using dye-diffused films and dye-blended films. Diffusion condition: $130^{\circ} \mathrm{C}, 5 \mathrm{~min}$. 

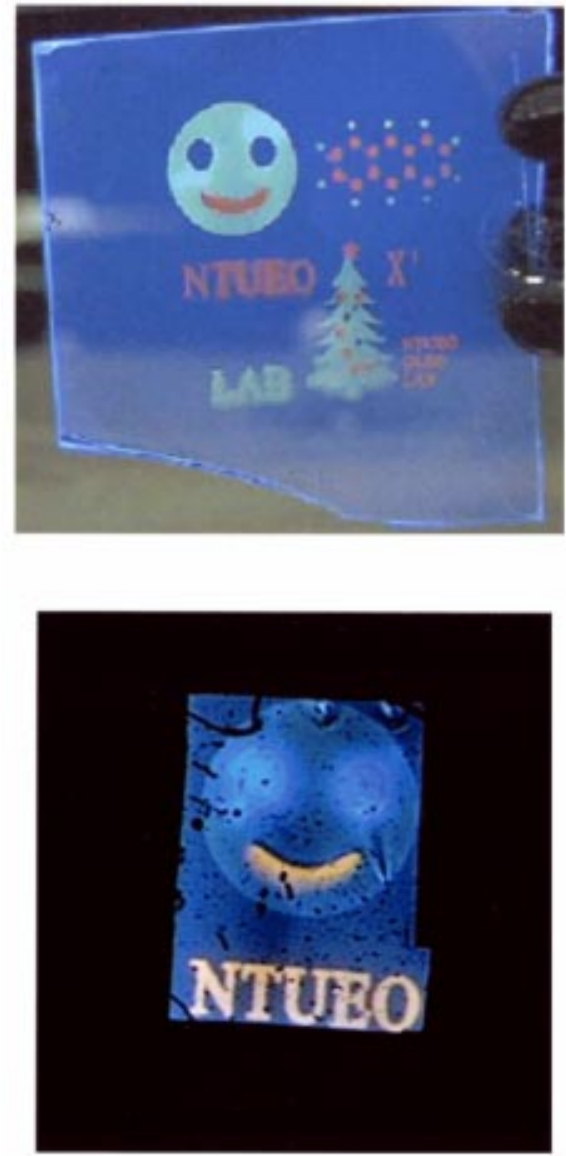

FIG. 5. (Color) (a) UV-excited PL image of a dye-diffused multicolor film. (b) EL image of a multicolor OLED (eyes: perylene; face: C6; mouth and letters: nile red).

that FS-D2T2 is a relatively effective and reliable technique for doping organic films.

By using a source plate containing color patterns, FSD2T2 could be used to accomplish color integration for OLED devices or displays on a substrate with a single thermal transfer step. The source plate containing multicolor dye-dispersed polymer patterns may be fabricated with any patterning technique, ranging from photolithography to printing, as long as the processing does not degrade the emission characteristics of dyes to be transferred. Screen printing, an additive and high-throughput patterning technique suitable for large areas, has been used as a demonstration. RGB inks were prepared by dispersing nile red, C6, and perylene into a commercial screen-printing resin paste (DuPont 7155) and were printed sequentially. Figure 5 shows the UV-excited PL image and the electroluminescence (EL) image of a PVK:PBD film receiving FS-D2T2, which clearly exhibits color-tuning dye patterns transferred from a screen-printed color source plate. Using a manual screen printer, we have been able to produce a linewidth of $\sim 150 \mu \mathrm{m}$ in the receiver film. Since, in the FS-D2T2 process, the diffusion length to obtain complete dye transfer is of the order of the receiver film thickness $(\sim 1000 \AA)$, lateral diffusion would not hinder attainment of $\sim \mu \mathrm{m}$ resolution eventually by reducing the feature sizes on the source plates.

Since the FS-D2T2 process relies on the diffusing capability of dyes in a polymer matrix, one may be concerned about the aggregation of dopant molecules and subsequent luminescence quenching during device operation. If this is an issue, surely one solution is to use dopant molecules less subject to molecular aggregation. ${ }^{13}$ On the other hand, it should be noted that the thermal transfer is performed at elevated temperatures, and that dye diffusion in a polymer matrix is a highly thermally activated process and heavily depends on the thermodynamic properties of the polymer matrix. ${ }^{14,15}$ For glassy polymers, dye-diffusing capabilities comparable to what are reported in this letter (i.e., PVK:PBD matrix) usually can only be obtained above their glass transition temperature $\left(T_{g}\right)$ and it drops exponentially below $T_{g} \cdot{ }^{14,15}$ For instance, our own thermal transfer experiments on pure PVK show that a temperature above $210^{\circ} \mathrm{C}\left(T_{g}\right.$ of $\mathrm{PVK}$ ) is necessary for a rapid transfer. Therefore, by using a matrix with a $T_{g}$ and a transfer temperature much higher than the operation temperature, the capability of dopant migration and aggregation during device operation should be effectively suppressed.

In conclusion, we have introduced FS-D2T2 as an effective method of performing controllable doping of polymer films in OLEDs. We show that this doping process can be modeled by Fick's diffusion theory. We applied this doping technique to color tuning of OLEDs without degrading device performance. Along with patterned color source plates, we have fabricated multicolor OLEDs of arbitrary patterns over large areas with a single thermal transfer step.

The authors would like to express gratitude to Professor T. Y. Luh, M. K. Leung, G. J. Jan, L. A. Wang, and H. C. Chang of NTU, Mr. H. R. Lien of Bayer Co., and Junsun Technology Co. for stimulating discussions and for assistance in experiments. This work was supported by National Science Council and Ministry of Education of the Republic of China (Grant No. NSC 88-2212-E-002-075 and 89-N$5 \mathrm{~A} 01-2-4-2)$.

${ }^{1}$ C. W. Tang and S. A. VanSlyke, Appl. Phys. Lett. 51, 913 (1987).

${ }^{2}$ J. H. Burroughes, D. D. C. Bradley, A. R. Brown, R. N. Marks, K. Mackay, R. H. Friend, P. L. Burns, and A. B. Holmes, Nature (London) 347, 539 (1990).

${ }^{3}$ C. C. Wu, J. C. Sturm, R. A. Register, and M. E. Thompson, Appl. Phys. Lett. 69, 3117 (1996).

${ }^{4}$ D. G. Lidzey, M. A. Pate, M. S. Weaver, T. A. Fisher, and D. D. C. Bradley, Synth. Met. 82, 141 (1996).

${ }^{5}$ T. R. Hebner, C. C. Wu, D. Marcy, M. H. Lu, and J. C. Sturm, Appl. Phys. Lett. 72, 519 (1998)

${ }^{6}$ T. R. Hebner and J. C. Sturm, Appl. Phys. Lett. 73, 1775 (1998).

${ }^{7}$ J. Bharathan and Y. Yang, Appl. Phys. Lett. 72, 2660 (1998).

${ }^{8}$ T. Shimoda, S. Kanbe, H. Kobayashi, S. Seki, H. Kiguchi, I. Yudasaka, M. Kimura, S. Miyashita, R. H. Friend, J. H. Burroughes, and C. R. Towns, Symposium Proceedings of the Society for Information Display, San Jose, CA (1999), p. 376.

${ }^{9}$ F. Pschenitzka and J. C. Sturm, Appl. Phys. Lett. 74, 1913 (1999).

${ }^{10}$ J. Crank, The Mathematics of Diffusion (Oxford University Press, Oxford, U.K. 1975).

${ }^{11}$ Chemical Technology in Printing and Imaging Systems, edited by J. A. G. Drake (Royal Society of Chemistry, Cambridge, U.K. 1993).

${ }^{12}$ R. A. Hann and N. C. Beck, J. Imaging Sci. Technol. 16, 238 (1990).

${ }^{13}$ B. X. Mi, Z. Q. Gao, C. S. Lee, S. T. Lee, H. L. Kwong, and N. B. Wong, Appl. Phys. Lett. 75, 4055 (1999).

${ }^{14}$ D. Ehlich and H. Sillescu, Macromolecules 23, 1600 (1990).

${ }^{15}$ P. F. Nealey, R. E. Cohen, and A. S. Argon, Macromolecules 26, 1287 (1993). 